\title{
Pallidal stimulation in dystonia: effects on cognition, mood, and quality of life
}

\author{
T D Hälbig, D Gruber, U A Kopp, G-H Schneider, T Trottenberg, A Kupsch
}

J Neurol Neurosurg Psychiatry 2005;76:1713-1716. doi: 10.1136/jnnp.2004.057992

Bilateral deep brain stimulation (DBS) of the globus pallidus internus (GPi) alleviates symptoms in patients with dystonia but its effects on cognition, neuropsychiatric status, and quality of life have not been examined. This is a case series report of 15 consecutive patients with different forms of dystonia who underwent bilateral implantation of DBS electrodes in the GPi. The patients were evaluated preoperatively and after 3-12 months of DBS with tests of cognition (Mattis Dementia Rating Scale, Stroop Test, Trail Making Test, Phonemic and Category Word Fluency, Digit Span, Rey Auditory Verbal Learning Test, Tonic and Phasic Alertness), neuropsychiatric status (Beck Depression and Anxiety Inventories, Montgomery Asberg Depression Rating Scale, Snaith-Hamilton Pleasure Scale, Brief Psychiatric Rating Scale), quality of life, and motor functions. GPi DBS significantly improved dystonic symptoms, functional abilities, and quality of life allowing for a significant reduction of antidystonic medications. No deterioration was observed in cognitive scores and neuropsychiatric measures. The present case series report thus provides preliminary evidence for the safety of GPi DBS regarding cognitive and neuropsychiatric functions in patients with dystonia.

$\mathrm{D}$ ystonia is a clinically heterogeneous motor syndrome characterised by sustained and often painful muscle contractions, frequently causing twisting, repetitive movements or abnormal postures. ${ }^{1}$ With the exception of dopa-responsive dystonia, pharmacological treatment is mostly unsatisfactory in generalised or multifocal dystonia.

Recently, several publications have reported beneficial effects of deep brain stimulation (DBS) of the internal globus pallidus (GPi) in primary and tardive dystonia. ${ }^{2-5}$ DBS is a reversible "non-lesioning" surgical treatment and is continuously applied via stereotactically implanted macroelectrodes. ${ }^{6}$ Interestingly, the neuropsychological effects of DBS of the GPi in dystonia are not known.

DBS of the GPi has been used for the treatment of Parkinson's disease (PD). Even if GPi DBS in PD in general seems to be safe regarding cognition, ${ }^{7}$ some studies have reported postoperative impairments of executive functions, verbal fluency, and memory (delayed free and cued recall). ${ }^{8} 9$ Furthermore, a recent study reported higher prevalence of suicide in patients treated with DBS for various movement disorders. ${ }^{10}$ Finally, total energy of GPi DBS used in dystonia, mainly pulse-width and voltage, are usually much higher than in PD, suggesting the possibility of neuropsychological side effects. ${ }^{11}$ The aim of the present case series report was therefore to assess the influence of bilateral GPi stimulation on cognition, neuropsychiatric status, and quality of life in dystonia.

\section{METHODS \\ Patients}

We included 15 (six male, nine female) consecutive patients with dystonia who were treated with bilateral GPi DBS. Of these, 13 patients had primary dystonia (three positive for torsion dystonia gene $(D Y T 1))$ and two patients had tardive dystonia according to the criteria recently published by Adityanjee and colleagues. ${ }^{12}$ Mean (SD) age of disease onset was 37.5 (20) years (range 6-62). At surgery, patients had a mean disease duration of 12.6 (9.6) years (range 2.5-40) and a mean age of 45.5 (17.7) years (range 13-68). Before surgery, all patients had marked disability due to segmental, multifocal, or generalised dystonia and had not achieved sufficient benefit from medical treatment including anticholinergics, tetrabenazine, pimozide, primidone, tetrazepam, lorazepam, clobazam, lithium, baclofen, and zolpidem. For additional patient characteristics see table 2 (available online at http://www.jnnp.com/supplemental/). Patients were considered for DBS when their medical status was stable and neuropsychiatric functioning allowed cooperation during the surgical procedure and postsurgical management of DBS devices. However, none of the patients who were screened during the enrolment period was rejected for DBS because of neuropsychological dysfunctions. All patients older than 18 years of age or the legal representatives of those patients younger than 18 years gave written informed consent before surgery.

\section{Target localisation}

We chose the primary stereotactic target point in the posteroventral medial globus pallidus at $20 \mathrm{~mm}$ lateral to the midline of the third ventricle, $4 \mathrm{~mm}$ below the intercommissural line (ICL), and $3 \mathrm{~mm}$ anterior to the midcommissural point similar to that used in patients with PD. ${ }^{13}$ Adjustments of the intended coordinates were made in accordance to the individual stereotactic MRI. Intraoperative electrode positions were adjusted according to clinical effects of macrostimulation in all patients and with reference to teleradiography by using the intraoperatively performed contrast ventriculography and a long distance biorthogonal $x$ ray system. If no optic or capsular responses were evoked during macrostimulation below a threshold of $2 \mathrm{~mA}(0.1 \mathrm{~ms}$ pulse duration, $130 \mathrm{~Hz}$ ), a permanent quadripolar electrode was inserted (model 3387; Medtronic, Minneapolis, MN). Furthermore, postoperative MRI was done to verify correct electrode placement and control for subclinical complications.

\section{Procedures and tests}

We assessed the patients before and a mean of 6.5 months (range 3-12) after surgery. Postsurgical testing was

Abbreviations: BFMDRS, Burke-Fahn-Marsden Dystonia Rating Scale; DBS, deep brain stimulation; GPi, globus pallidus internus; PD, Parkinson's disease; TMT, Trail Making Test 
Table 1 Presurgical and postsurgical scores for motor functions, quality of life, neuropsychiatric status, and cognition $(n=15)$ and number of patients demonstrating changes $>1$ and $>2 S D$

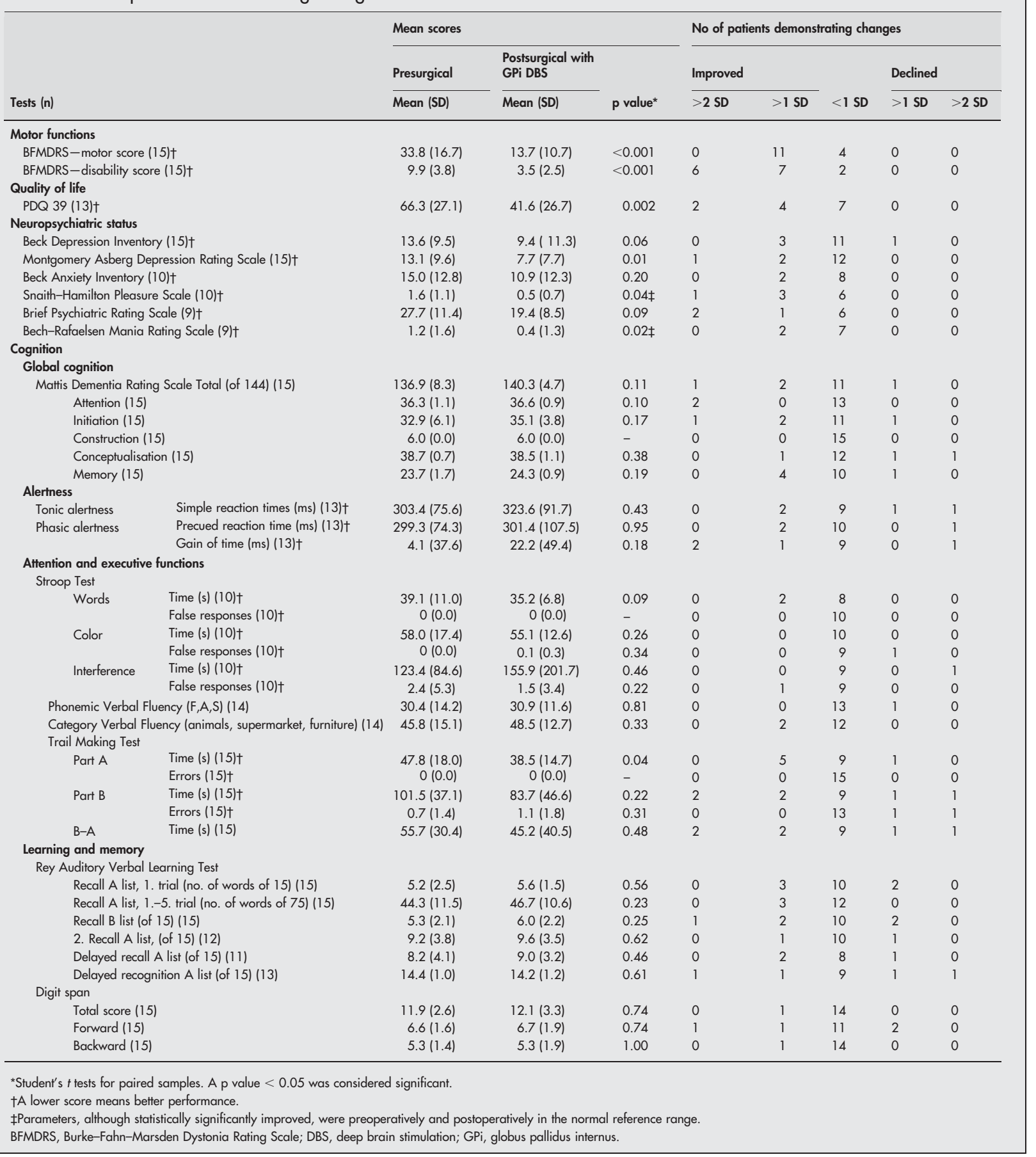

conducted while patients were on stimulation with DBS parameters that were individually optimised for each patient. Patients were evaluated on their medications. To test the clinical efficacy of DBS, we evaluated the dystonic symptoms with the Burke-Fahn-Marsden Dystonia Rating Scale (BFMDRS), ${ }^{14}$ which assesses symptom severity and disability in functional activities. For a global cognitive evaluation we used the Mattis Dementia Rating Scale (DRS), ${ }^{15}$ and to evaluate alertness, patients performed simple and precued visual reaction tasks. ${ }^{16}$ As tests of attention and executive functions, patients had to perform the Stroop Test, ${ }^{17}$ Phonemic and Category Verbal Fluency tasks, ${ }^{17}$ and the
Trail Making Test (TMT). ${ }^{17}$ Memory functions were evaluated using parallel versions of the Rey Auditory Verbal Learning Test (RAVLT) ${ }^{17}$ and the Digit span test. ${ }^{17}$ Mood and neuropsychiatric state were assessed using the Montgomery Asberg Depression Rating Scale (MADRS), ${ }^{18}$ the Beck Depression Inventory (BDI), ${ }^{19}$ the Beck Anxiety Inventory (BAI), ${ }^{20}$ the Snaith-Hamilton Pleasure Scale (SHAPS), ${ }^{21}$ the Brief Psychiatric Rating Scale (BPRS), ${ }^{22}$ and the BechRafaelsen Mania Rating Scale (BMRS). ${ }^{23}$ Since no specific scale for the assessment of quality of life in patients with dystonia was available, the PDQ-39, ${ }^{24}$ a questionnaire originally designed for PD was administered. 


\section{Data analysis}

Student's $t$ tests for paired samples were calculated for comparison of presurgical and postsurgical test scores. Because we aimed to assess the safety of a clinical intervention, a magnified type 1 error was appropriate and no correction for multiple comparisons was made.

\section{RESULTS}

There were no lasting adverse events associated with electrode implantation. At the time of the postoperative evaluation, stimulator settings using an anodal case and one or two adjacent cathodal contacts were as follows:

- Mean (SD) voltage:

- right GPi-3.1 (1.2) V (range: 1.5-5.0)

- left GPi-3.1 (1.1) V (range 1.5-5.0)

- Mean pulse width:

- both sides-106.0 (55.4) $\mu$ s (range: 60.0-210.0)

- Mean frequency:

- both sides-142.3 (32.2) Hz (range: 130.0-250.0).

In 10 patients the antidystonic medications could be decreased or tapered off (see table 2B online at http:// www.jnnp.com/supplemental/).

Motor functions as assessed by the BFMDRS motor score improved in all patients (range 26-93\%; table 2B). Accordingly, group analysis showed a significant mean improvement of dystonic symptoms by approximately $60 \%$ (table 1), whereas BFMDRS disability scores improved post surgically by about $65 \%$.

Quality of life improved in all patients and resulted in a significant overall improvement of 37\% (PDQ-39; table 1). Apart from a slight improvement on part A of the TMT (TMTA; table 1), there was no significant effect on any of the cognitive tests. However, descriptive analysis revealed that individual patients showed improvement or decline on single or several test measures (see table 3 online at http:// www.jnnp.com/supplemental/). For example, patient 8 remained stable on 26 cognitive measures and consistently worsened on five measures whereas patient 11 was stable on 19 measures and consistently improved on 11 measures. On neuropsychiatric tests, the only significant effect of GPi DBS was a mild improvement in depression. Remaining neuropsychiatric scores were unchanged or improved within the normal reference range (tables 1 and 3; for table 3 see online at http://www.jnnp.com/supplemental/).

\section{DISCUSSION}

Dystonic symptoms in our patients were reduced postsurgically by almost $60 \%$. This confirms efficient pallidal electrode placement and complies with previous reports on beneficial effects of GPi DBS on motor symptoms in dystonia. ${ }^{2-5}$ Furthermore, stimulation parameters in our patients were similar to parameters reported by other studies. ${ }^{24}$

Overall, we found cognitive measures stable or mildly improved. The only significant effect of stimulation was an improvement on TMT-A. The TMT-A is thought as baseline for the cognitively rather complex part B of the TMT. Since the TMT-A heavily relies on motor processes, the decrease in performance time on stimulation is likely to be attributable to the overall improvement of motor function. However, cognitive changes with deteriorations and improvements were found in individual patients. These changes could not be related to preoperative patient characteristics. Therefore, our data does not allow identification of predictors for postsurgical cognitive outcome.

The marked improvement of motor symptoms allowed a postsurgical reduction or cessation of antidystonic medication in 10/15 patients. Since most antidystonic drugs are known to potentially impair cognition, we cannot entirely rule out that postsurgical medication changes masked neuropsychological deterioration induced by GPi DBS. Indeed non-parametrical post hoc comparisons of performance in those patients with unchanged medication regimen and patients with reduced antidystonic medication showed that simple reaction times after surgery increased in patients with stable medication. However, all remaining cognitive and neuropsychiatric parameters were unchanged. More importantly, non-parametrical post hoc analysis revealed that the five patients who were tested before and after surgery under identical pharmacological treatment conditions did not change on any of the cognitive measures.

Subjects were repeatedly tested and for many established cognitive tests, no parallel versions are available. In some instances, therefore, the same tests had to be applied twice. Thus, test-retest effects may have offset DBS induced cognitive deterioration. However, there are no test-retest effects described for both the tonic and phasic alertness tests. ${ }^{16}$ Furthermore, for the RAVLT, parallel versions were used. Although reliability measures of the remaining cognitive tests are considered reasonably good, ${ }^{17}$ practise effects have been described for the DRS, TMT, Stroop, and Fluency tasks. We therefore cannot rule out the possibility that test-retest effects may have masked mild cognitive decline on some of the cognitive measures.

With the exception of mild improvement on depression scales, neuropsychiatric status including anxiety, anhedonia, and mania did not change. The finding of mildly improved mood is most likely secondary to marked alleviation of disabling dystonic symptoms and improvement of functional abilities.

Although after surgery our patients were not assessed without DBS and our study does not allow differentiation between effects of surgery (micropallidotomy) and DBS, the present study provides preliminary support for the safety of GPi DBS in dystonia with regard to cognition, mood, and neuropsychiatric status. This result is compatible with recent neuroanatomical research suggesting a neuroanatomical and functional subdivision of the GPi with posteroventrolateral parts mediating motor functions and mediodorsal parts cognitive functions. ${ }^{25}$ Since DBS of the GPi targets posteroventral parts of the GPi, our observation of improved motor function and unaffected neuropsychological status is consistent with the dissociation of motor and non-motor parts of the GPi. ${ }^{25}$

In summary, the present case series report supports the efficacy of bilateral pallidal stimulation in the treatment of dystonia. More importantly, it is the first report providing preliminary support for the cognitive and neuropsychiatric safety of GPi DBS. Finally, it has been demonstrated that GPi DBS leads to an overall improvement of functional abilities and quality of life in a condition which can be most disabling. Our preliminary results will have to be confirmed by long term studies involving larger and more homogeneous patient samples differentiating between different age groups, retesting of dystonic control patients who do not undergo surgery, and using even more comprehensive neuropsychological test batteries.

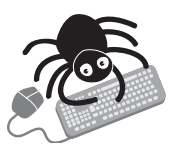

An appendix to this paper has been posted online at http://www.jnnp.com/supplemental/ 


\section{Authors' affiliations}

T D Hälbig, D Gruber, U A Kopp, T Trottenberg, A Kupsch, Department of Neurology, Charité-University Medicine Berlin, Berlin, Germany G-H Schneider, Department of Neurosurgery, Charité-University Medicine Berlin, Berlin, Germany

Competing interests: none declared

Correspondence to: Dr T D Hälbig, Department of Neurology, Mount Sinai School of Medicine, One Gustave L Levy Place, Box 1137, New York, NY 10029, USA; thomas.haelbig@mssm.edu

Received 3 November 2004

Revised version received 7 March 2005

Accepted 25 March 2005

\section{REFERENCES}

1 Fahn S, Bressman SB, Marsden CD. Classification of dystonia. Adv Neurol 1998;78: 1-10

2 Krauss JK, Yianni J, Loher TJ, et al. Deep brain stimulation for dystonia. J Clin Neurophysiol, 2004;21, 18-30.

3 Damier $\mathbf{P}$, Thobois S, Witjas T, et al. Treatment of tardive dyskinesia by pallidal stimulation: results from the French multicenter study STARDYS. Neurology 2004;62:A504.

4 Trottenberg T, Volkmann J, Deuschl G, et al. The treatment of severe tardive dystonia with pallidal deep brain stimulation. Neurology 2005;64:344-6.

5 Vidailhet M, Vercueil L, Houeto JL, et al. Bilateral deep-brain stimulation of the globus pallidus in primary generalized dystonia. N Engl J Med 2005;352:459-67.

6 Benabid AL, Benazzouz A, Hoffmann D, et al. Long-term electrical inhibition of deep brain targets in movement disorders. Mov Disord 1998;13(Suppl 3): $119-25$

7 Ardouin C, Pillon B, Peiffer E, et al. Bilateral subthalamic or pallidal stimulation for Parkinson's disease affects neither memory nor executive functions: a consecutive series of 62 patients. Ann Neurol 1999:46:217-23.

8 Jahanshahi M, Ardouin CM, Brown RG, et al. The impact of deep brain stimulation on executive function in Parkinson's disease. Brain 2000;123(Pt 6):1142-54.
9 Troster Al, Fields JA, Wilkinson SB, et al. Unilateral pallidal stimulation for Parkinson's disease: neurobehavioral functioning before and 3 months after electrode implantation. Neurology 1997;49:1078-83.

10 Burkhard P, Vingerhoets FP, Berney A, et al. Death by suicide after deep brain stimulation. Movement Disorders 2004;19:S316.

11 Coubes P, Roubertie A, Vayssiere N, et al. Treatment of DYT1-generalised dystonia by stimulation of the internal globus pallidus. Lancet 2000;355:2220-1

12 Adityanjee, Aderibigbe YA, Jampala VC, et al. The current status of tardive dystonia. Biol Psychiatry 1999:45:715-30.

13 Starr PA. Placement of deep brain stimulators into the subthalamic nucleus or globus pallidus internus: technical approach. Stereotact Funct Neurosurg 2002; 79: 1 18-45.

14 Burke RE, Fahn S, Marsden CD, et al. Validity and reliability of a rating scale for the primary torsion dystonias. Neurology 1985;35:73-7.

15 Mattis S. Mental status examination for organic mental syndrome in the elderly patient. In: Bellak L, Karasu TB, eds. Geriatric Psychiatry. New York: Grune \& Stratton, 1976

16 Zimmermann P, Fimm B. TAP-Test for Attentional Performance, German Version 1.6. Herzogenrath, Germany: Psychologische Testsysteme V, Fimm, 2001.

17 Lezak M. Neuropsychological Assessment. New York: Oxford University Press, 1995.

18 Montgomery SA, Asberg M. A new depression scale designed to be sensitive to change. Br J Psychiatry 1979;134:382-9.

19 Hautzinger M, Keller F, Worall H, et al. Beck-Depressions-Inventar (BDI), 2nd edn., vol. Bern: Huber, 1995.

20 Markgraf J, Ehlers A. Beck Angstinventar, Deutsche Version (BAl). Göttingen: Hogrefe, 2002

21 Snaith RP, Hamilton M, Morley S, et al. A scale for the assessment of hedonic tone the Snaith-Hamilton Pleasure Scale. Br J Psychiatry 1995;167:99-103.

22 Overall JE, Gorham DR. The Brief Psychiatric Rating Scale, Psychol Rep, 1962:799-812.

23 Bech P, Rafaelsen OJ, Kramp P, et al. The mania rating scale: scale construction and inter-observer agreement. Neuropharmacology 1978;17:430-1.

24 Peto V, Jenkinson C, Fitzpatrick R. PDQ-39: a review of the development, validation and application of a Parkinson's disease quality of life questionnaire and its associated measures. J Neurol 1998;245(supp 1):S10-S14.

25 Middleton FA, Strick PL. Anatomical evidence for cerebellar and basal ganglia involvement in higher cognitive function. Science 1994;266:458-61. 